\title{
Performance Evaluation of ADV DSR and God in VANET for City and Highway Scenario
}

\author{
Richa Sharma ${ }^{1}$ and Dr. Jyoteesh Malhotra ${ }^{2}$ \\ ${ }^{1,2}$ CSE Department Guru Nanak Dev University Regional Campus Jalandhar \\ Punjab, India \\ ${ }^{1}$ rixs11111991@gmail.com,Jyoteesh@gmail.com²
}

\begin{abstract}
Vehicular Ad-Hoc network has taken the enormous attention from various researchers due to its vast applications like traffic management, electronic toll collection, parking lot payment, multimedia and many more. Vehicular Ad-Hoc network is an emerging network. It is a special type of Intelligent Transport System which provides an improved vehicle to vehicle and vehicle to interface communication and inform the vehicles about road and dynamic traffic conditions so as to provide efficient data dissemination among the vehicles. Routing protocols play a vital role in communication among entities which exchanges information to establish a route, decision in forwarding and action in maintaining the route or recovering from routing failure. While analyzing traditional routing protocols for MANETs it is demonstrated that their performance is poor in VANET. So there is frequent need of performance evaluation in real scenarios. The work done in this paper has considered ad-hoc nodes to test the performance of routing protocols in city and highway scenario. In doing so, the performance evaluation has been done here in terms of quality of service parameters like throughput ratio, packet drop rate and collision rate using exhaustive simulations.
\end{abstract}

Keywords: MANET, ADV, DSR, GOD \& NCTUns6.0

\section{Introduction}

With the tremendous increase in number of vehicles, people are facing problems like traffic congestion. The congestion and relevant vehicle accommodation problems, leads to constant amount of accidents. Lacking of road traffic safety leads to threat to human lives and also poses danger to our environment as well. Initial precautions like seat belts and airbags are not sufficient enough to eliminate problems arising due to driver's inability to see the situation ahead of time. The desire to facilitate the passengers and increase safety of road users was the main motive behind the development of Vehicular Ad-Hoc Network. VANET is a new technique of communication among vehicles even at high speeds on roads which uses dedicated short range radio signals DSRC $(5.9 \mathrm{GHz})$ standard which employs the IEEE $802.11 \mathrm{p}$ for wireless vehicular communication. VANET integrates multiple networking technologies such as Wi-Fi IEEE 802.11p, WAVE IEEE 1609, WiMAX IEEE 802.16, Bluetooth, IRA and ZigBee. [3]VANET deploys services like traffic management, electronic toll collection, traffic engineering, and provides emergency information, parking lot payment and many more. VANET are basically similar to MANET but high mobility, varying node density, high processing power, movement at high speeds, difficult communication scenarios with short link lifetime are the various characteristics which differ it from MANET. Due to varying network topologies and its dynamic nature routing problems becomes more challengeable and complex. Routing protocols in VANET should be reliable, adaptable to network load, robust and must have minimum latency. VANET deploys factors like lack of information 
about node's neighbors, short network duration and lack of global area network for routing protocols [9].

The objective of this paper is to analyze the MANET routing protocols in realistic VANET environment by using reliable simulator having both network and traffic simulation capabilities like NCTUns6.0 which provides solution to simulate IEEE 802.11(p) vehicular networks. Mostly many researchers focuses on using single environment of VANET to evaluate the performance of various routing protocols but in our research both highway and city scenario are used to determine which protocol gives better performance using QOS parameters.

This paper is organized as follows. Related work is described in section II. Section III describes the various MANET based routing protocols to be evaluated. Simulative methodology is described in section IV. Section V describes the result evaluation and finally section VI concludes the paper.

\section{Related Work}

VANET prototyping in real time environment is a challenging task. Many researchers have been deployed in last few years yet there is need of proper systematic performance evaluation and comparison of MANET based routing protocols to be used in VANET realistic environment.

Singh. P et al., [1] evaluated the performance of VANET using QOS metrics for AODV, DSR and ADDMR (Adaptive Demand Driven Multicast Routing) multicast protocols in urban environment. The QOS performance is evaluated by using metrics like routing overhead, average delay with 1024 bytes of packets, packet delivery ratio and concluded that with the increase in number of nodes average delay for protocols also increases but DSR has less delay than others and also overhead is very less. Also we concluded that AODV performance is less but it reduces excessive memory requirement and helps in route redundancy. ADDMR also gives best results according to the paper as it adopts the change of mobility and control periodic unnecessary messages.

Omer masood et al., [4] evaluates that VANET has a really changing topology, also triggered partial updates are more effective than periodic updates which help to increase throughput. Simulation results shows that ADV outperforms AODV in terms of audio and video data transmission, also $\mathrm{ADV}$ has less routing overheads and packet loss than AODV responds quickly to network load variations and also throughput of ADV is higher. This paper concludes that with increase in network load more packets use the channels this leads to increase in collision and route discovery in AODV takes so long. On the other hand, ADV efficiently distributes the routing information and also minimizes the number of routing packets used thus leaving more bandwidth for data packets.

Shailkul at al., [3] compared the performance of various reactive routing protocols like DSR, AODV, AOMDV in VANET by using different mobility model in VANET MobiSim framework and changing the parameters like mobility, node speed, number of sources using different performance metrics. On mobility model two types of graphs were generated user defined and space graph, later performance metrics shows that performance of AOMDV is better but performance decreases in space graph model. It is concluded that AOMDV has drawback as it requires more routing load.

Until now few studies [5-7] have compared various traditional ad-hoc routing protocols in VANET environment using NS2 simulator. Simulations done in VANET are very hostile and do not deploy realistic VANET scenarios. Simple topologies on roads were considered in maximum cases, so to investigate these protocols we need a simulator that have both network and traffic simulation. NCTUns6.0 is such a tool having both network and traffic simulation and thus to validate our finding we use this simulator for simulative investigation of protocols in both highway and city scenario in VANET. 


\section{Routing Protocols}

In the past few years routing in VANET is widely studied and investigated. As VANET belong to special class of Ad-Hoc networks, the traditionally used Ad-Hoc routing protocols for MANET have been evaluated and tested to be used in VANET environment. Using of location based and topology based routing protocols of MANET requires the assigning of unique addresses to participating nodes but in VANET environment these protocols cannot take guarantee to avoid the allocation of duplicate addresses among vehicles in the network. Due to various routing related issues it has prompted researchers to find such routing algorithms that are more scalable, robust and can deliver improved packet delivery ratio and better throughput.

Furthermore in location based protocols there is frequent need for location services and servers so our main focus later is on topology based routing protocols as they have not these mechanisms and very useful in simulative investigation in VANET environment. Following are some of the protocols to be chosen for simulative investigation in different traffic scenarios:

- DSR: Dynamic Source Routing is basically used for wireless mesh networks and like AODV these are on-demand routing protocols. DSR reduces overheads on a network and uses source routing mechanism instead on depending on routing tables at each intermediate node. It keeps a cache to store source routes learned by the nodes. Here whenever a valid route from source to destination is not present in route cache route discovery is initiated by source.

- ADV: Adaptive Distance Vector is based on distance vector algorithm which implies sequence number to solve the Long Lived Loop problem. The main feature of this routing protocol is overhead reduction and helps the originating nodes to know the freshest route track. Control packets are used here to maintain the route table of each node. Whenever there is update ADV does not trigger the change of metric for a new routing entry, trigger meter keeps update of assigning valid route.

- GOD: General Operation Director Algorithm is used to store global information regarding the state of nodes, network and environment. In simulation GOD is defined to manage the details of operations like movement patterns.

\section{Simulator Methodology}

"Simulation is the technique of solving problems by the analyzing the performance in virtual environment of a model known as simulator system". Simulation development is an iterative process in which the analysis, construction and execution of a model repeatedly perform in order to achieve desired level of understandings. This section starts with providing a framework and selection of simulation tool, then setup and various parameters used in simulation. Afterwards the simulation scenario is used. 


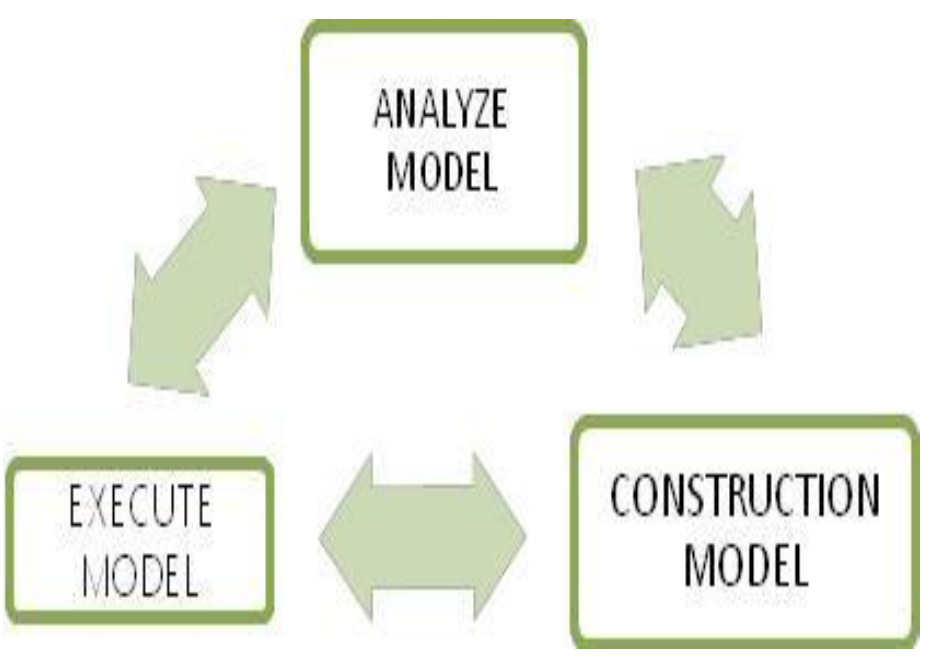

Figure 1. A Simulation Process

\subsection{Simulator Selection:}

Simulation is a technique of solving problems by analyzing the performance in virtual environment of a model known as simulator. A number of simulations exist for VANET but none of them have been up to the mark and none of them can provide a completion solution set for simulating VANET. From the traffic simulator perspective, the traces generated once seem useless after a certain time as the dynamics of traffic change abruptly. Another problem that remains difficult to solve is the inter communication issue between the two simulators i.e., traffic and network simulator. Without a solid solution to this problem, the inter communication between the two still remains a matter of discussion. Many simulators of VANET like Groove Sim, Highway Traffic Safety Application, Fleet Net, CARLINK, Car2Car, NHTSA (National Highway Traffic Safety Application), IP PReVENT, NCTUns are used to do perform simulation in VANET. Still these issues have been resolved by NCTUns (National Chiao Tung University Network Simulator), which is a GUI based integrated network and traffic simulator and emulator that implies kernel re-entering method for simulation results. NCTUns can simulate technologies like 802.11a, 802.11b, 802.11g and 802.11p. RED-HAT LINUX supported NCTUns with Fedora12 and C++ language purely written. NCTUns have features like:

- It includes free space with shadowing path loss model.

- Uses TCP/IP stack for packet passing.

- Supports maximum of 4096 nodes inside single simulation.

- Overcomes the drawbacks of MOVE, TraNS and VANET Mobisim.

- Repeatable and faster simulation results

\subsection{Performance Parameters}

To check the performance of routing protocols different parameters are used in highly mobile environment of VANET. In our study performance of various routing protocols can be compared based on the three parameters namely Throughput, Packet Drop and collision rate as follows. 
- Throughput

The average number of successful delivered data packets on a network node describes throughput. In NCTUns simulation results of throughput shows the sum of received packets at destination in $\mathrm{Kb} / \mathrm{sec}$. It is calculated in bytes/sec or data packets per second. Throughput $($ Bytes/sec $)=\sum$ (Number of received packets at destination $) *$ Packet size

\section{- Packets Drop}

$$
\sum \text { Simulation Time }
$$

The total number of packets not successfully reach to destination describes packet drop. Congestion, queue overflow and traffic can be major reasons behind packet drop. Higher protocol performance shows lower packer drops.

- Collision rate

Collision rate implies as number of packets collides per second. Collision rate should be less for better performance.

\subsection{Simulation Scenario}

A city scenario and highway scenario are considered here with variable number of nodes (vehicles) and variable speed.

The scenario is drawn with "draw topology" feature of NCTUns-6.0 simulator. Then using "edit topology" feature various performance parameters are set according to the requirements as shown in table below:

Table I. Specification of Performance Parameters

\begin{tabular}{|c|c|c|}
\hline SNO. & PARAMETERS & VALUE \\
\hline 1. & Frequency & 2400(MHz) \\
\hline 2. & Transmission power & $15(\mathrm{dbm})$ \\
\hline 3. & Antenna gain & 1 \\
\hline 4. & Link bandwidth & $11(\mathrm{Mbps})$ \\
\hline 5. & Speed & $100(\mathrm{~ns})$ \\
\hline 6. & Mobility Model & Manhatten Grid \\
\hline
\end{tabular}

After it the simulations are done using "run" interface available in toolbar of NCTUns6.0 workspace. 


\section{Case1. City Scenario}

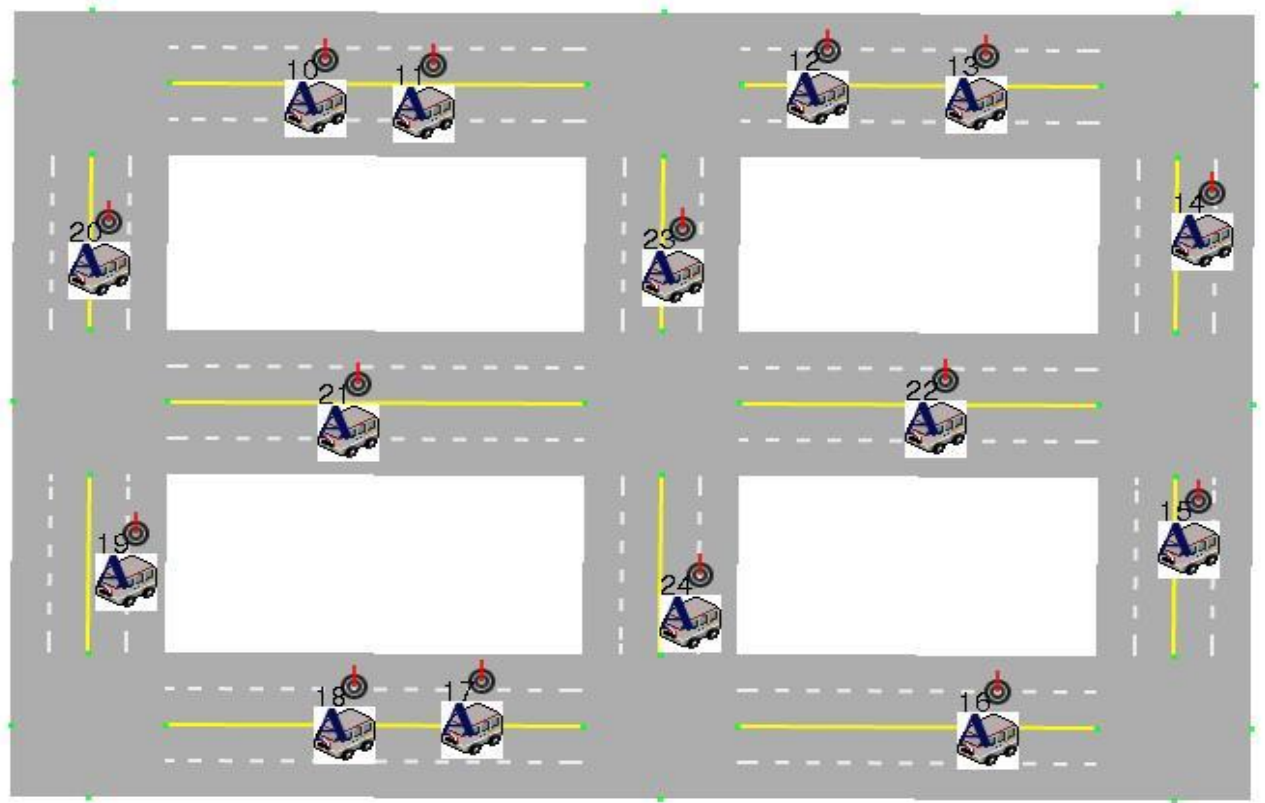

Figure 2. City Scenario Case with 15 Nodes

\section{Case 2 Highway scenario}

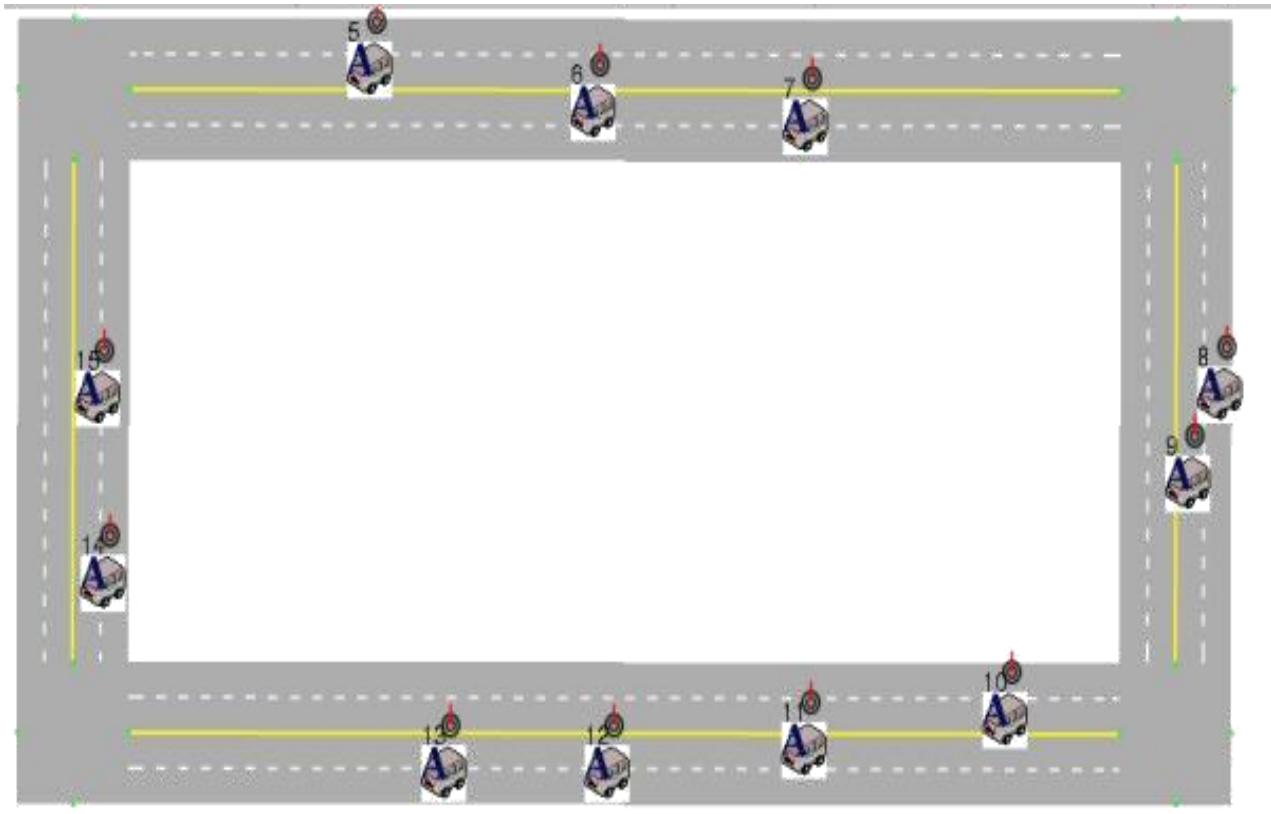

Figure 3. Highway Scenario Case with 11 Nodes

\section{Result and Analysis}

The graph listed below shows the performance of UDP protocols in both the scenarios. These graphs are based on following the performance parameters:

- Throughput rate

- Packet drop rate

- Collision rate 
Case 1 City Scenario

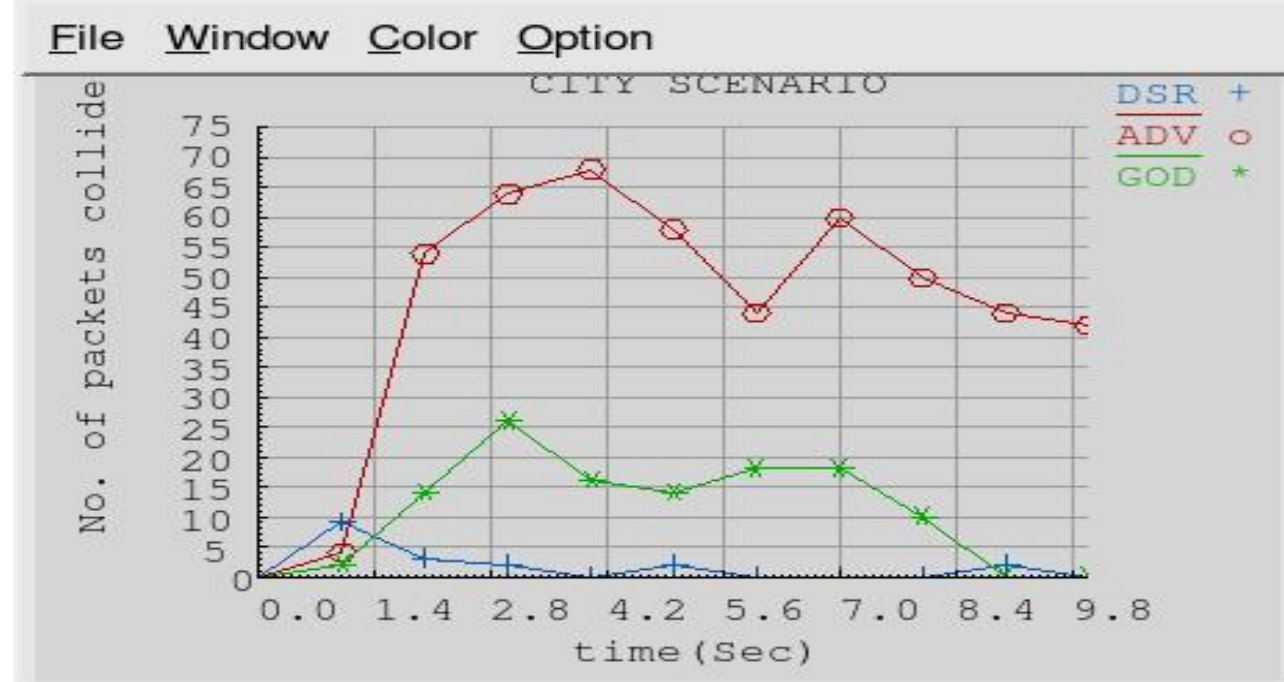

Figure 4. Collision Rate for Different Routing Protocols in City Scenario File Window Color Opption

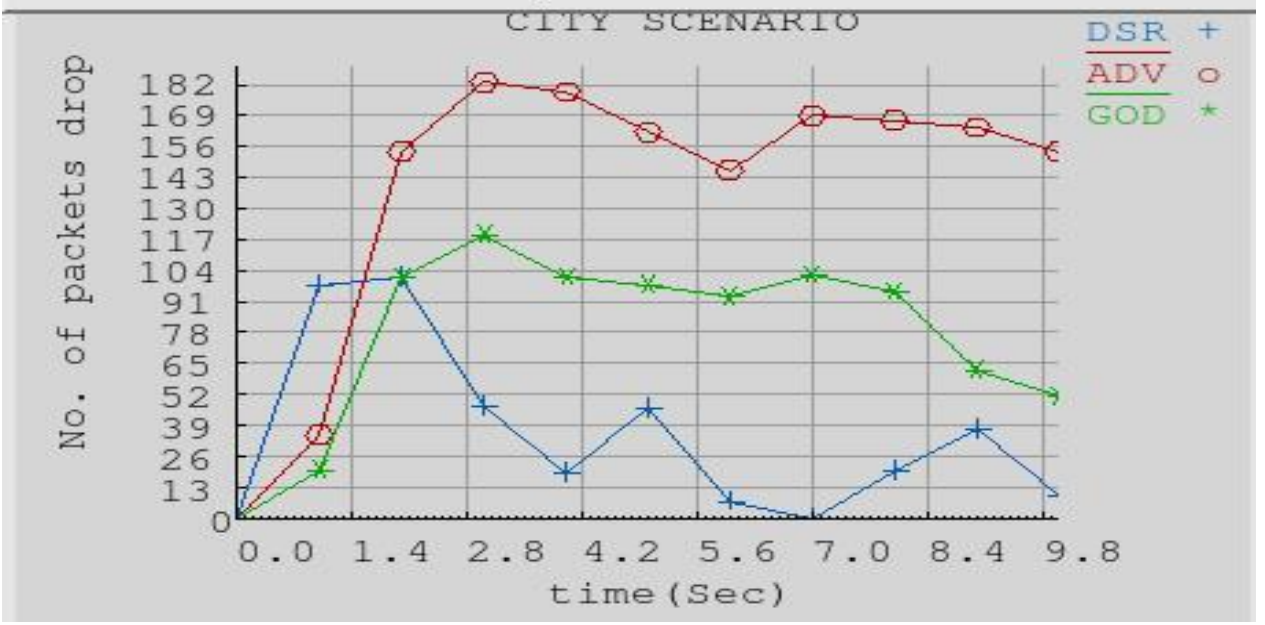

Figure 5. Packet Drop Rate for Different Routing Protocols in City Scenario

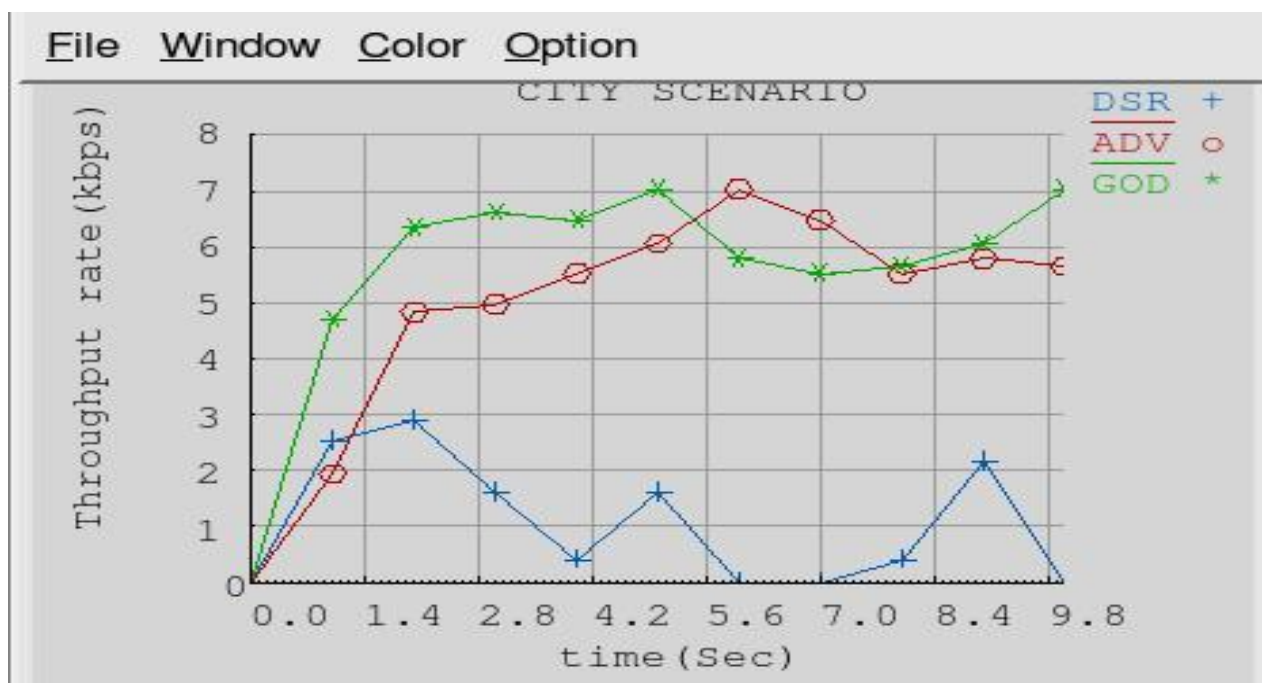

Figure 6. Throughput Rate for Different Routing Protocols in City Scenario 
Case 2 Highway scenario

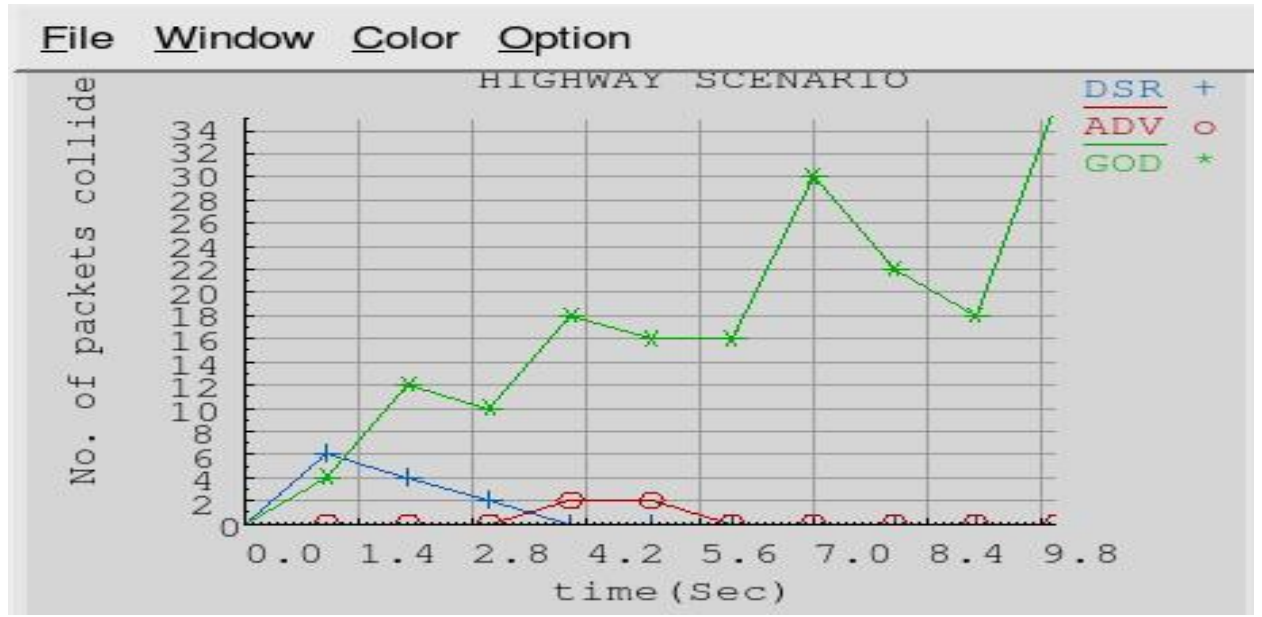

Figure 7. Collision Rate for Different Routing Protocols in Highway Scenario

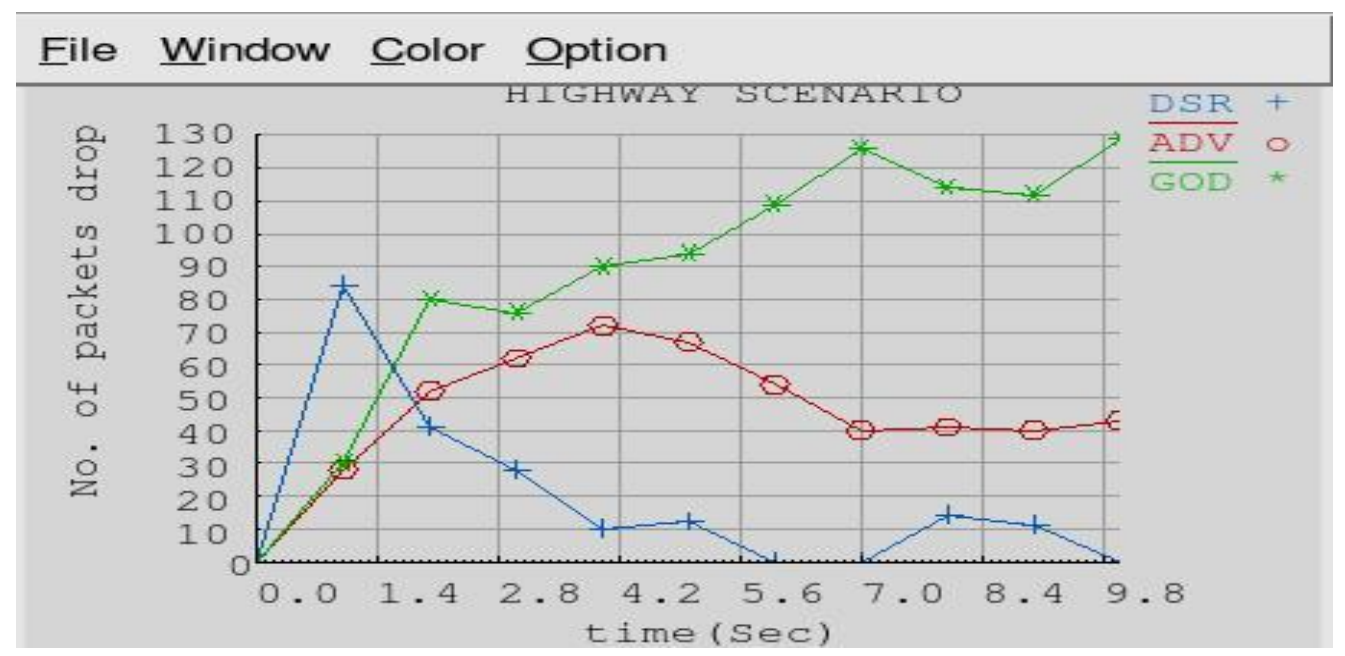

Figure 8. Packet Drop Rate for Different Routing Protocols in Highway Scenario

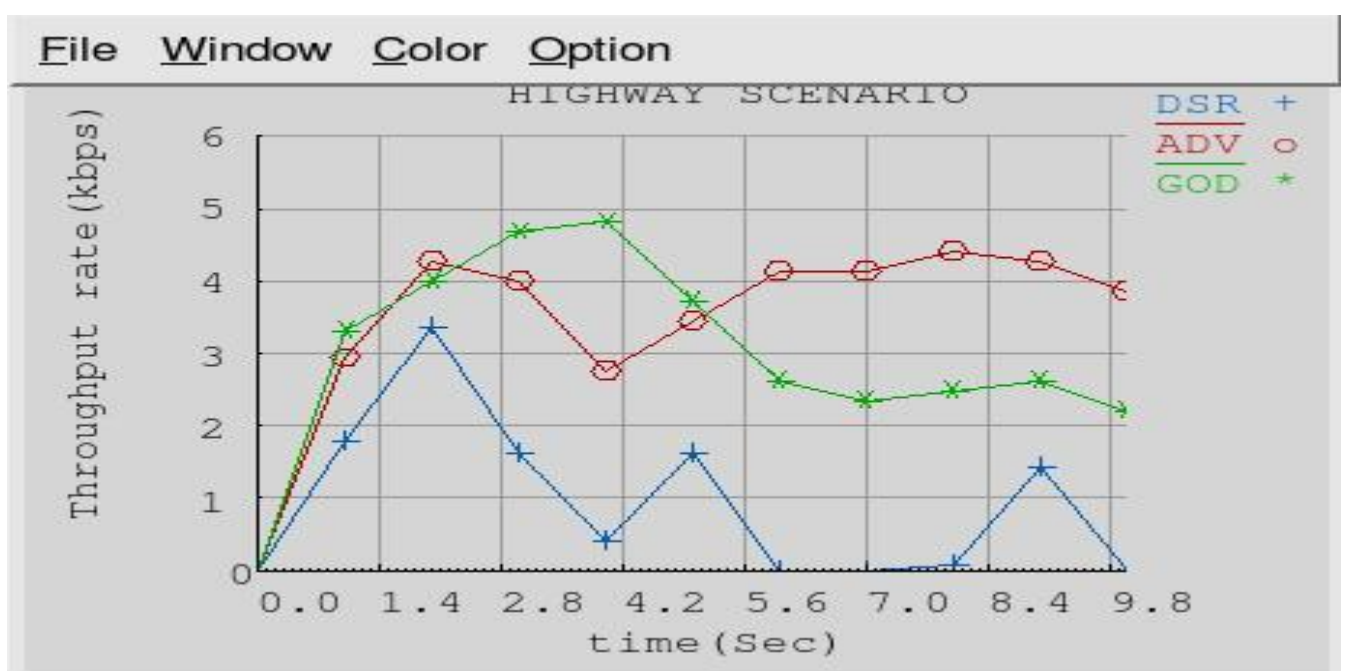

Figure 9. Throughput Rate for Different Routing Protocols in Highway Scenario 
The quality of service parameters (throughput and packet drop) has been evaluated over the time interval of 20 seconds for city and highway scenario and has been provided in tabular form as given below:

Table 2. Performance Evaluation in City Scenario

\begin{tabular}{|c|c|c|c|}
\hline CITY SCENARIO & ADV & DSR & GOD \\
\hline Throughput(kbps) & 6.9 & 3 & 7 \\
\hline Collision rate & 182 & 103 & 117 \\
\hline Packet drop rate & 185 & 102 & 120 \\
\hline
\end{tabular}

Table 3. Performance Evaluation in Highway Scenario

\begin{tabular}{|c|c|c|c|}
\hline $\begin{array}{l}\text { HIGHWAY } \\
\text { SCENARIO }\end{array}$ & ADV & DSR & GOD \\
\hline Throughput(kbps) & 4.5 & 3.2 & 4.9 \\
\hline Collision rate & 2 & 6 & 32 \\
\hline Packet drop rate & 70 & 82 & 130 \\
\hline
\end{tabular}

It can be analyzed from the table that throughput ratio is high in GOD and low in DSR routing protocol for city scenario. The collision rate is high in ADV while minimum for DSR routing protocol while the packet drop rate is maximum for ADV and minimum for DSR routing protocol for city scenario. While for the highway scenario maximum throughput is given by GOD and minimum throughput is provided by the DSR. The collision rate of GOD is maximum and minimum for ADV. The packet drop rate of GOD is maximum and minimum for ADV for highway scenario. Thus, overall performance indicates that $\mathrm{ADV}$ is best suitable for highway scenario while GOD is best suitable for city scenario.

\section{Conclusion}

The main goal of this paper is to identify the performance of different routing protocols ADV, DSR and GOD in terms of different parameters which effect the performance of VANET and to evaluate quality of service parameters (throughput and packet drop) in city as well as highway scenario and it has been found that ADV is best suitable for highway scenario while GOD is best suitable for city scenario under free space network conditions. It can be used as reference for the active researchers in this field. In this paper effect of different types of routing protocols in VANET has been examined. It has been found that quality of service in city scenario (in terms of collision rate) is less than highway scenario because of network congestion. The performance issues regarding path connectivity and time delay should be improved. It is hoped that this paper will provide guidelines for active researchers working on routing protocols in the VANET. 


\title{
References
}

[1] P. Singh, "Comparative study between unicast and multicast routing protocols in different data rates using VANET", IEEE International conference on issues and challenges in 2014, IEEE publications, (2014) February 7-8, pp. 278-284.

[2] J. Kaur and P. Singh, "Performance Comparison Between Unicast \& Multicast Protocols in Vanet", International Journal of Advanced Technology \& Engineering Research (IJATER), vol. 3, no. 1, (2013) January, pp. 109-115.

[3] S. Islam Chowdhury, W.-I. Lee, Y.-S. Choi, G.-Y. Kee and J.-Y. Pyun, "Performance Evaluation of Reactive Routing Protocols in VANET", 17th Asia-Pacific Conference on Communications (APCC) IEEE 2nd - 5th October 2011 Sutera Harbour Resort, Kota Kinabalu, Sabah, Malaysia, pp. 559-564.

[4] O. Masood, A. Akram and M. Nadeem Majeed, "Performance Evaluation of ADV with AODV for Realtime and Multimedia Applications in Vehicular Ad-hoc Networks (VANETs)", IEEE Communication society, vol. 17, no. 3, (2013) March.

[5] J. Nzounta, N. Rajgure, G. (Grace) Wang and C. Borcea, "VANET Routing on City Roads Using Realtime Vehicular Traffic Information", IEEE Transactions On Vehicular Technology, vol. 58, no. 7, (2009) September.

[6] K. Dalal, P. Chaudhary and P. Dahiya, "Performance Evaluation of TCP and UDP Protocols in VANET Scenario using NCTUns-6.0 simulation tool”, IJCA, vol. 36, no. 6, (2011) December.

[7] P. Chaudhary, P. Dahiya and K. Dalal, "Performance Evaluation of AODV And ADV Protocols In VANET Scenario", Int. J.Comp.Tech. Appl, vol. 3, no. 1, pp. 50-55.

[8] B. Vidhale, S. S. Dorle, "Performance Analysis of Routing Protocols in Realistic Environment for Vehicular Ad Hoc Network", $21^{\text {st }}$ International conference on Systems Engineering (ICSE), IEEE Conference Publications, (2013), pp. 267-272.

[9] S. Saha, Dr. U. Roy and Dr. D. D. Sinha, "VANET Simulation in Different Indian City Scenario", Advance in Electronic and Electric Engineering, ISSN 2231-1297, vol. 3, no. 9, (2013), pp. 1221-1228.

[10] R. Kumar, "A Comparative Study of Various Routing Protocols in VANET", IJCSI International Journal of Computer Science Issues, vol. 8, Issue 4, no. 1, (2011) July, pp. 643-684.

[11] B. Paul, Md. Ibrahim and Md. A. Naser Bikas, "VANET routing Pros and Cons", International Journal of Computer, vol. 20, no. 3, (2011) April.

[12] S. A. Ade and P. A. Tijare, "Performance Comparison of AODV, DSDV, OLSR, DSR Routing protocols in MANET", International Journal of Information Technology and Knowledge Management, vol. 2, no. 2, (2010) July-December, pp. 545-548.

[13] A GUI based user manual for NCTUns6.0 simulator.

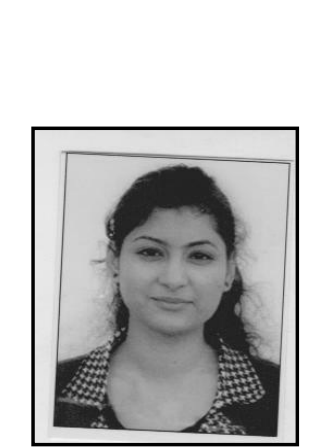

\begin{abstract}
Authors
Richa Sharma received her B.Tech degree in Computer Science and Engineering from Punjab College of Engineering \& Technlogy, Punjab, India in 2012, pursing her M.Tech degree in Computer Science and Engineering with specialization in Vehicular Ad- Hoc Network from Guru Nanak Dev University, Amritsar, India. Her research interests include wireless communication and vehicular area networks.
\end{abstract}

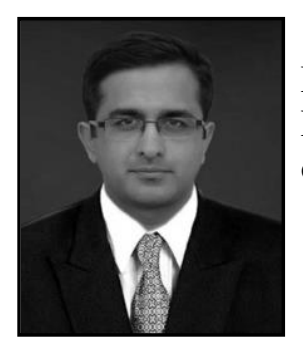

Dr Jyoteesh Malhotra, B.Engg.(with Distinction), M.Tech (Gold Medalist), PhD (ECE), Head of CSE and ECE department Guru Nanak Dev University Regional Campus Jalandhar, INDIA. His area of Specialization is Wireless / Optical Comm. Systems and Networks. 